\title{
Investigation of Cyclobenzaprine Interactions with P450 Cytochromes CYP1A2 and CYP3A4 through Molecular Docking Tools
}

\author{
Douglas Vieira Thomaz ${ }^{1}$, Edson Silvio Batista Rodrigues ${ }^{1}$, Fábio Bahls Machado ${ }^{1}$, \\ Isaac Yves Lopes de Macedo ${ }^{1}$ \\ ${ }^{1}$ Universidade Federal de Goiás \\ 240 street, Leste Universitário district, Goiânia - G0, 74605-170, Brazil
}

DOI: $10.22178 /$ pos.43-1

LCC Subject Category: RM1-950

Received 20.01.2019

Accepted 20.02.2019

Published online 28.02.2019

Corresponding Author:

Douglas Vieira Thomaz

douglasvthomaz@gmail.com

(C) 2019 The Authors. This article

is licensed under a Creative Commons

Attribution 4.0 License @) (1)
Abstract. Cyclobenzaprine (CBP) is a centrally acting muscle relaxant whose myriad of therapeutic applications imply the need of better understanding its pharmacokinetics and thermodynamics. Henceforth, this work was concerned with an in silico investigation of CBP main metabolizers in the human organism, namely CYP1A2 and CYP3A4. For this purpose, computational methods were employed, such as molecular docking and other semi-empirical approaches. Results evidenced that the model herein depicted for CBP-CYP1A2 may not reproducibly represent the physiological interaction between CBP and this enzyme. Moreover, CBP-CYP3A4 docking results evidence thermodynamic feasibility of the molecular docking model and were further corroborated by literature, what may reproducibly represent a possible interaction between CBP and this macromolecule.

Keywords: tricyclic; cheminformatics; redox enzymes; metabolism; pharmacokinesis.

\section{INTRODUCTION}

Cyclobenzaprine (CBP) is a centrally acting muscle relaxant whose applicability range from treating acute musculoskeletal pain to chronic conditions such as fibromyalgia. The structure of this compound is quite similar to that of tricyclic antidepressants, which implies the possibility of yet unknown therapeutic uses. Albeit CBP metabolism has been studied under the light of different methods over the years, the thermodynamics and kinetics of its molecular docking interactions in the context of xenobiotic metabolism are yet unknown [4, 5, 22, 24].

CBP metabolism is primarily mediated by $\mathrm{P} 450$ family cytochromes (CYP), more specifically CYP1A2 and CYP3A4, what implies that the association of this muscle relaxant with other drugs primarily metabolized by these CYP isoforms might result in important interactions, which could henceforth affect patient safety. Although the enzymes responsible for CBP metabolism have been identified, there were, to the best of our knowledge, no studies regarding the thermodynamics of CBP-CYP interaction [17, 19, 20, 23].
Regarding molecular docking strategies, programs such as AutoDock Vina allows fast scoring function set up as well as quick calculations, what is optimal in preliminary screenings for pharmacodynamical prediction as well as medicinal chemistry studies. Furthermore, the concomitant employment of semi-empirical approaches to minimize the energy of complex chemical compounds, further increase the appeal of computational chemistry in investigations concerning drug-receptor interactions $[1,6,7]$.

In view of the importance of better understanding CBP metabolism as well as the thermodynamics of its docking with the major metabolizers (CYP1A2 and CYP3A4), this work is intended to employ cheminformatics tools to establish a semi-flexible docking model of CBP-CYP interaction. Henceforth, CBP structure had its energy minimized through molecular mechanic functions and was subjected to docking studies as a flexible ligand to interact with a rigid macromolecule i.e. CYP. 


\section{METHODS}

CBP (dimethyl(3-\{tricyclo[9.4.0.0 $\left.0^{3},{ }^{8}\right]$ pentadeca1(15),3,5,7,9,11,13-heptaen-2-ylidene\}propyl)amine) structure was minimized through Chimera software version 1.13 coupled to Molecular Modeling Toolkit (MMTK) and AMBER toolkit 4.0 prior docking studies. The same software was used to edit protein units retrieved from Protein DataBank (PDB). Moreover, the software Phyton Molecular Viewer (PMV) version 1.5.6 was used to evaluate torsion-prone regions in CBP molecule, and the docking models were conducted using AutoDock Vina and AutoDock Tools version 1.5.6. The docking model herein employed is based on a flexible ligand and a rigid receptor, therefore configuring itself in a semi-flexible model $[2,16$, 18]. The minimization in energy conformation and HOMO surface calculations were performed employing the MM2 Force Field in ChemDraw3D®Pro 12.

CYP1A2 (PDB entry: 2HI4) and CYP3A4 (PDB entry: 4NY4) were used in this study.

Concerning intermolecular thermodynamics, Gibbs free energy values correlate to the maximum of reversible work feasible at a given system. In this context, it can be related to the inter- action proneness between two molecules in a docking model. This parameter can be calculated through the following equation (1):

$$
\Delta G=R T \ln K_{i},
$$

where $\Delta G$ is the interaction affinity; $R$ is the gas constant and $T$ is the temperature.

The equation (1) can be derived into order to yield the interaction constant $K_{i}$.

$$
K_{i}=e^{\frac{\Delta G}{R T}}
$$

where $e$ is the Euler's number.

\section{RESULTS AND DISCUSSION}

In order to prepare CBP molecule for docking, CBP energy was minimized henceforth rendering the lowest energy conformer. Moreover, the highest occupied molecular orbital (HOMO) regions were also calculated as well as the torsionfeasible regions. Results are displayed in Figure 1.
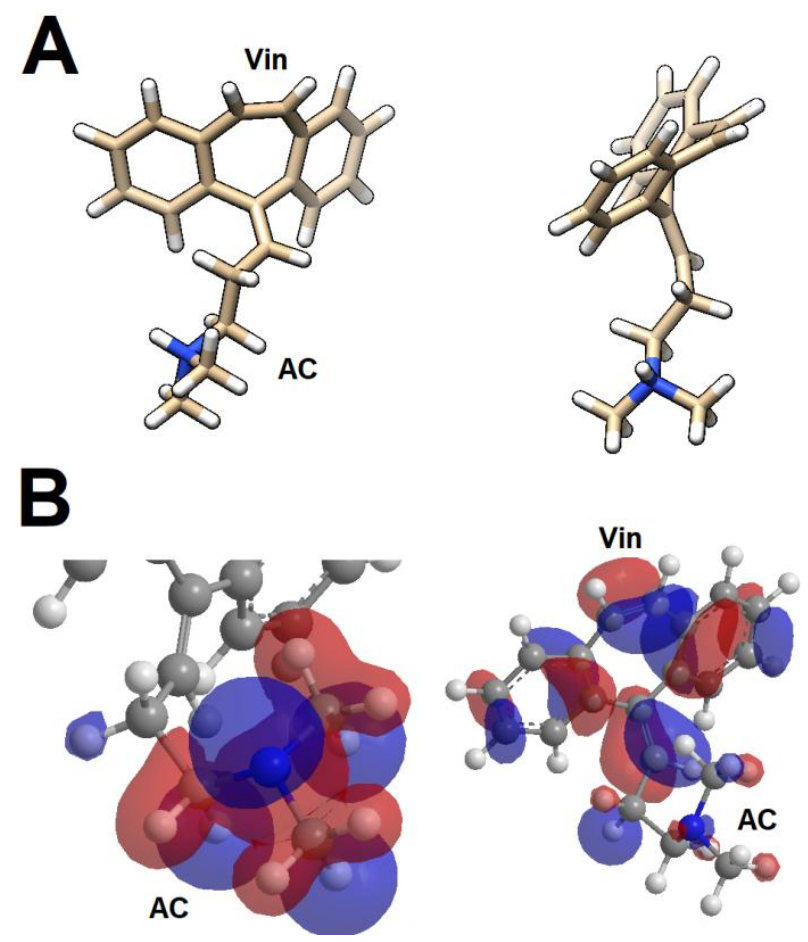

Figure 1 - Results of CBP energy minimization

Notes: A) Chemical structure of CBP lowest energy conformer evidencing a vinylic bridge (Vin) and the terminal aliphatic chain (AC). B) HOMO depictions of CBP lowest energy conformer depicting low (Blue) and high (Red) electron density regions. AC region correlates to HOMO-1 whereas Vin correlates to HOMO.

All data gathered through Chimera software version 1.13. 
Results evidenced that CBP lowest energy conformer tricyclic ring does display a slight distortion in the vinyl moiety (Vin) towards the same plane of the terminal aliphatic chain (AC), which grants the molecule a bent shape (Figure 1A). Moreover, HOMO regions of the molecule evidence that there are two sites of elevated electron density in CBP, namely the Vin moiety and the tri-substituted amine in the AC. Further analysis evidenced that these moieties correlate to HOMO-1 and HOMO in Vin and AC, respectively (Figure 1B). Concerning torsion-prone re- gions, CBP AC is the sole moiety capable of rotation hence it is based on $\sigma^{\text {sp3-sp3 }}$ covalent bonds.

A molecular docking study was undergone to analyze CBP interaction with CYP1A2. Henceforth the gridline for the docking study was set around the protoporphyrin ring of CYP1A2 molecule employing high exhaustiveness, which was set to 20. Figure 2 displays the highest scoring model with the distances between CBP, nearby residues and the iron moiety in the protoporphyrin ring system measured in angstroms $(\AA)$.

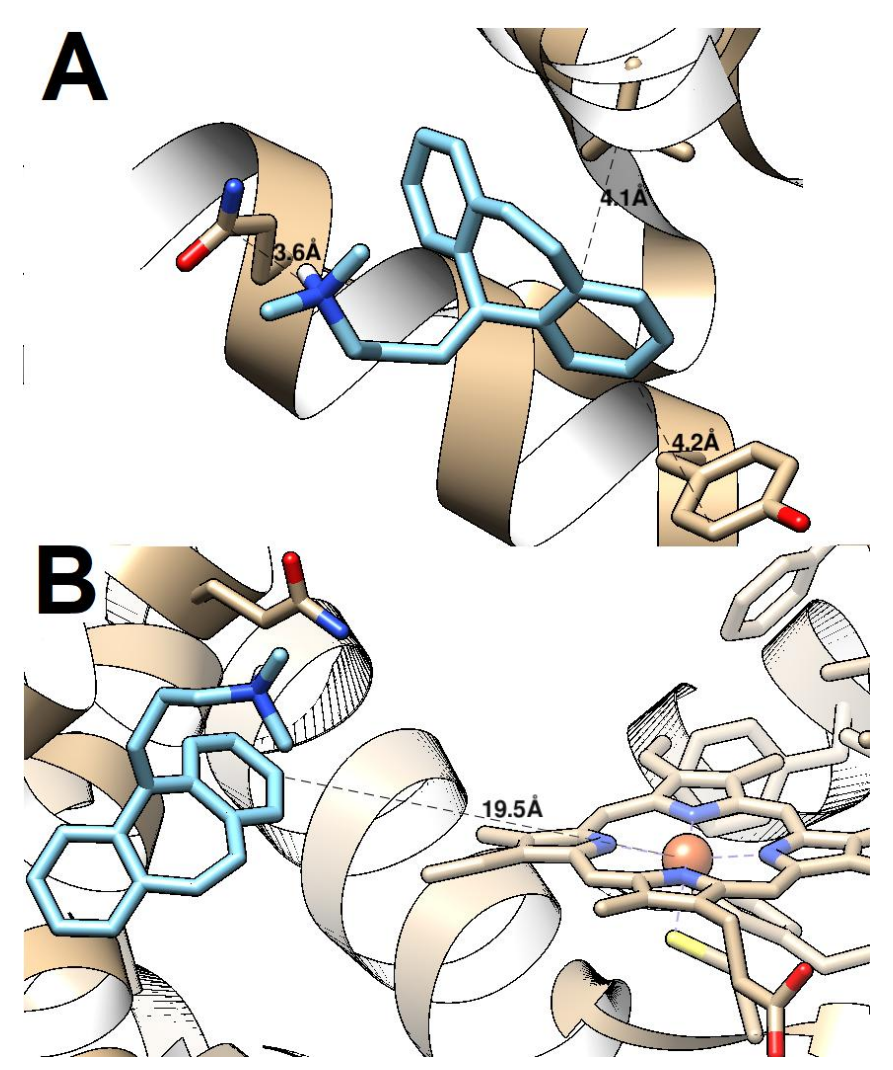

Figure 2 - Docking depiction of the highest scoring model for CBP-CYP1A2 interaction

Notes: NDistances therein highlighted between CBP molecule (Blue) and nearby residues as well as to the iron moiety of the protoporphiric group (orange sphere). All data gathered through Chimera software version 1.13.

CBP highest scoring docking models presented no hydrogen bonds, albeit dipole interactions were seen (Figure 2A), which is a common occurrence in docking models $[8,9,10]$. However, the distance between CBP and the iron moiety in the protoporphyrin ring is nonetheless too distant to seemingly promote catalysis $(19.5 \AA)$. In this context, the model herein presented for CBPCYP1A2 interaction may not be able to efficiently correlate itself with experimental data reported in the literature $[11,12,15,25]$.
Although some models scored below the best presented additional hydrogen bonds, which are usually responsible for thermodynamic stable interactions between molecules, non-specific dipole interactions; molecular packaging and steric hindrance may generate false positives in docking models $[3,13,14,21]$. Given that the prosthetic group of the macromolecule herein concerned is intimately involved in reactional steps, the model; although thermodynamic feasible, may not be reproducible $[12,15]$. 
CBP interactions with CYP3A4 were also evaluated through a molecular docking. Henceforth the gridline for the docking study was again set around the protoporphyrin ring; this time; of the CYP3A4 molecule. Furthermore, exhaustiveness was set to 20. Figure 3 displays the highest scoring model with the distances between CBP and the iron moiety in the protoporphyrin ring system measured in angstroms $(\AA)$.

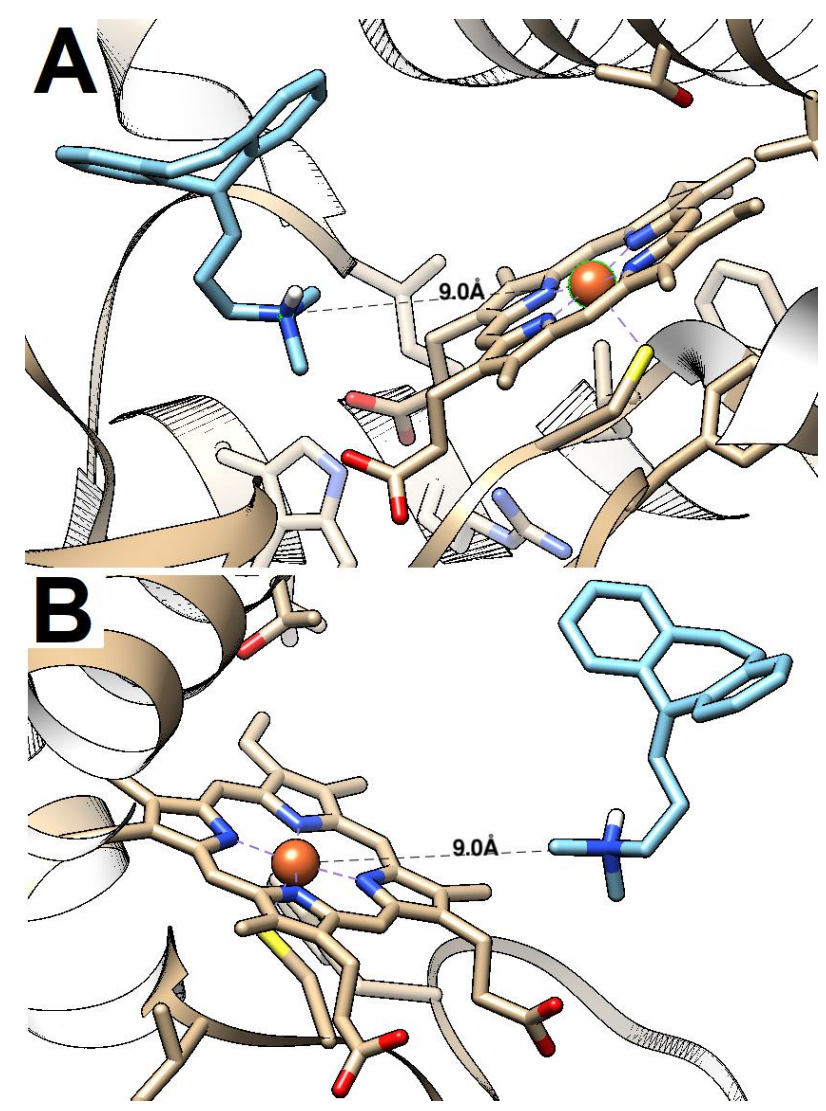

Figure 3 - Docking depiction of the highest scoring model for CBP-CYP3A4 interaction

Notes: Distances therein highlighted between CBP molecule (Blue) and the iron moiety of the protoporphiric group (orange sphere). All data gathered through Chimera software version 1.13.

Results evidenced that the highest scoring model presented optimal thermodynamic properties, and the distance between CBP and the iron moiety in the protoporphyrin ring system $(9.0 \AA)$ was nonetheless inferior to the one observed in the model above-displayed for CBP-CYP1A2 interaction (Figure 3A and 3B).

Literature reports that CYP catalytic properties are attributed to the protoporphyrin ring which is chelated to an iron unit. This moiety is the main prosthetic group of P450 enzymes, and the condensed pyrrole aromatic nuclei associated to the transition metal; which is itself a lewis acid; enhances henceforth the redox capabilities of these catalytic biomolecules. This effect is caused by the dislodgement of electrons from the ligand towards CYP-prosthetic group, what nonetheless further justifies the model herein depicted since the interacting molecule should be in close proximity to the prosthetic group for this process to happen $[12,15]$.

Equations (1) and (2) cited in the methods section were employed to calculate $K_{i}$ of the highest ranking docking models for CBP interaction with CYP1A2 and CYP3A4. Results are displayed in Table 1.

Results evidenced that $\mathrm{Ki}$ for CYP3A4 macromolecule interaction with CBP was higher than that of CBP-CYP1A2 interaction. These findings suggest therefore that both $\Delta G$ and $K_{i}$ values corroborate to higher thermodynamic feasibility of the CBP-CYP3A4 pose herein studied. 
Table 1 - Thermodynamic properties calculated for the lowest energy conformation in the docking of CBPCYP1A2 and CBP-CYP3A4

\begin{tabular}{|c|c|c|c|c|c|c|}
\hline Receptor & $\begin{array}{c}\text { Affinity } \\
\left(\mathrm{kcal}^{\mathrm{mol}}{ }^{-1}\right)\end{array}$ & 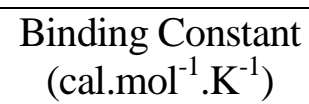 & $\begin{array}{l}\text { RMSD } \\
\text { l.b. }\end{array}$ & $\begin{array}{l}\text { RMSB } \\
\text { u.b. }\end{array}$ & $\begin{array}{l}\text { HBonds } \\
\text { (ligand) }\end{array}$ & $\begin{array}{l}\text { HBonds } \\
\text { (receptor) }\end{array}$ \\
\hline CYP1A2 & -8.0 & 0.9866 & 0.0 & 0.0 & 0 & 0 \\
\hline CYP3A4 & -8.8 & 0.9853 & 0.0 & 0.0 & 0 & 0 \\
\hline
\end{tabular}

\section{CONCLUSION}

This work presented a study regarding CBP interaction with CYP1A2 and CYP3A4. Results evidenced that the model herein depicted for CBPCYP1A2 may not reproducibly represent the physiological interaction between CBP and this enzyme. Moreover, CBP-CYP3A4 results from evidence thermodynamical feasibility of the molecular docking model and were further corroborated by literature, what may reproducibly represent a possible interaction between CBP and this macromolecule.

\section{REFERENCES}

1. Amaro, R. E., Baudry, J., Chodera, J., Demir, Ö., McCammon, J. A., Miao, Y., \& Smith, J. C. (2018). Ensemble Docking in Drug Discovery. Biophysical Journal, 114(10), 2271-2278. doi: 10.1016/j.bpj.2018.02.038

2. Case, D. A., Cheatham, T. E., Darden, T., Gohlke, H., Luo, R., Merz, K. M., ... Woods, R. J. (2005). The Amber biomolecular simulation programs. Journal of Computational Chemistry, 26(16), 16681688. doi: $10.1002 /$ jcc.20290

3. Cosconati, S., Forli, S., Perryman, A. L., Harris, R., Goodsell, D. S., \& Olson, A. J. (2010). Virtual screening with AutoDock: theory and practice. Expert Opinion on Drug Discovery, 5(6), 597-607. doi: $10.1517 / 17460441.2010 .484460$

4. Darwish, H. W., Naguib, I. A., \& Darwish, I. A. (2018). Five modified classical least squares based models for stability indicating analysis of cyclobenzaprine $\mathrm{HCl}$ with its major degradation products: A comparative study. Spectrochimica Acta Part A: Molecular and Biomolecular Spectroscopy, 204, 598-602. doi: 10.1016/j.saa.2018.06.101

5. De Santana Santos, T., Calazans, A. C. M., Martins-Filho, P. R. S., Silva, L. C. F. da, de Oliveira e Silva, E. D., \& Gomes, A. C. A. (2011). Evaluation of the muscle relaxant cyclobenzaprine after third-molar extraction. The Journal of the American Dental Association, 142(10), 1154-1162. doi: 10.14219/jada.archive.2011.0084

6. García-Nieto, J., López-Camacho, E., García-Godoy, M. J., Nebro, A. J., \& Aldana-Montes, J. F. (2019). Multi-objective ligand-protein docking with particle swarm optimizers. Swarm and Evolutionary Computation, 44, 439-452. doi: 10.1016/j.swevo.2018.05.007

7. Gupta, M., Sharma, R., \& Kumar, A. (2018). Docking techniques in pharmacology: How much promising? Computational Biology and Chemistry, 76, 210-217. doi: 10.1016/j.compbiolchem.2018.06.005

8. Jiang, X., Tsona, N. T., Tang, S., \& Du, L. (2018). Hydrogen bond docking preference in furans: O H $\cdots \pi$ vs. O H ‥ 0. Spectrochimica Acta Part A: Molecular and Biomolecular Spectroscopy, 191, 155-164. doi: 10.1016/j.saa.2017.10.006

9. Khorasani, R., \& Fleming, P. E. (2016). On calculating HR bond enthalpies using computational data. Computational and Theoretical Chemistry, 1096, 89-93. doi: 10.1016/j.comptc.2016.09.033

10. Kumar, S. P. (2018). PLHINT: A knowledge-driven computational approach based on the intermolecular $\mathrm{H}$ bond interactions at the protein-ligand interface from docking solutions. Journal of Molecular Graphics and Modelling, 79, 194-212. doi: 10.1016/j.jmgm.2017.12.002 
11. Lynch, D. E., \& Reeves, C. R. (2019). Statistical analysis of the effect of a single O H hydrogenbonding interaction on carbonyl bond lengths. Journal of Molecular Structure, 1180, 158-162. doi: 10.1016/j.molstruc.2018.11.100

12. Mena-Ulecia, K., \& MacLeod-Carey, D. (2018). Interactions of 2-phenyl-benzotriazole xenobiotic compounds with human Cytochrome P450-CYP1A1 by means of docking, molecular dynamics simulations and MM-GBSA calculations. Computational Biology and Chemistry, 74, 253-262. doi: 10.1016/j.compbiolchem.2018.04.004

13. Meng, X.-Y., Zhang, H.-X., Mezei, M., \& Cui, M. (2011). Molecular Docking: A Powerful Approach for Structure-Based Drug Discovery. Current Computer Aided-Drug Design, 7(2), 146-157. doi: $10.2174 / 157340911795677602$

14. Morris, G. M., \& Lim-Wilby, M. (2008). Molecular Docking. Molecular Modeling of Proteins, 365-382. doi: 10.1007/978-1-59745-177-2_19

15. Ramesh, M., \& Bharatam, P. V. (2014). Importance of hydrophobic parameters in identifying appropriate pose of CYP substrates in cytochromes. European Journal of Medicinal Chemistry, 71, 15-23. doi: 10.1016/j.ejmech.2013.10.023

16. Salomon-Ferrer, R., Case, D. A., \& Walker, R. C. (2012). An overview of the Amber biomolecular simulation package. Wiley Interdisciplinary Reviews: Computational Molecular Science, 3(2), 198210. doi: 10.1002/wcms.1121

17. Share, N. N., \& McFarlane, C. S. (1975). Cyclobenzaprine: A novel centrally acting skeletal muscle relaxant. Neuropharmacology, 14(9), 675-684. doi: 10.1016/0028-3908(75)90091-x

18. Trott, O., \& Olson, A. J. (2009). AutoDock Vina: Improving the speed and accuracy of docking with a new scoring function, efficient optimization, and multithreading. Journal of Computational Chemistry, NA-NA. doi: 10.1002/jcc.21334

19. Wang, R. W., Liu, L., Cheng, H. (1996). Identification of human liver cytochrome P450 isoforms involved in the in vitro metabolism of cyclobenzaprine. Drug Metabolism and Disposition, 24(7), 786-791.

20. Winchell, G. A., King, J. D., Chavez-Eng, C. M., Constanzer, M. L., \& Korn, S. H. (2002). Cyclobenzaprine pharmacokinetics, including the effects of age, gender, and hepatic insufficiency. The Journal of Clinical Pharmacology, 42(1), 61-69.

21. Wu, M.-Y., Dai, D.-Q., \& Yan, H. (2012). PRL-dock: Protein-ligand docking based on hydrogen bond matching and probabilistic relaxation labeling. Proteins: Structure, Function, and Bioinformatics, 80(9), 2137-2153. doi: 10.1002/prot.24104

22. Yang, Y. W., Macdonald, J. B., Nelson, S. A., \& Sekulic, A. (2017). Treatment of vismodegib-associated muscle cramps with cyclobenzaprine: A retrospective review. Journal of the American Academy of Dermatology, 77(6), 1170-1172. doi: 10.1016/j.jaad.2016.12.017

23. Zhang, D., Evans, F. E., Freeman, J. P., Yang, Y., Deck, J., \& Cerniglia, C. E. (1996). Formation of mammalian metabolites of cyclobenzaprine by the fungus, Cunninghamella elegans. ChemicoBiological Interactions, 102(2), 79-92. doi: 10.1016/s0009-2797(96)03736-2

24. Zhang, T.-T., Xue, R., Wang, X., Zhao, S.-W., An, L., Li, Y.-F., ... Li, S. (2018). Network-based drug repositioning: A novel strategy for discovering potential antidepressants and their mode of action. European Neuropsychopharmacology, 28(10), 1137-1150. doi: 10.1016/j.euroneuro.2018.07.096

25. Zhao, H., Tang, S., \& Du, L. (2017). Hydrogen bond docking site competition in methyl esters. Spectrochimica Acta Part A: Molecular and Biomolecular Spectroscopy, 181, 122-130. doi: 10.1016/j.saa.2017.03.038 